\title{
Intercomparaison et validation de différents modèles de propagation de houle en baie de Douarnenez.
}

\author{
David Morellato $^{1,2}$, Nicolas Guillou ${ }^{1,2}$, Georges Chapalain ${ }^{2}$, Eric \\ Duvieilbourg ${ }^{2}$ et Guy Amis ${ }^{1}$ \\ ${ }^{1}$ CETMEF - Technopôle Brest-Iroise - BP 5 - 29280 Plouzané, \\ France David.Morellato@equipement.gouv.fr, \\ Nicolas.guillou@equipement.gouv.fr,Guy.amis@equipement.gouv.fr \\ ${ }^{2}$ UMR CNRS-UBO 6539 LEMAR - Institut Universitaire Européen \\ de la Mer - Place Nicolas Copernic - 29280 Plouzané, France \\ David.Morellato@univ-brest.fr,Nicolas.Guillou@univ-brest.fr. \\ Georges.Chapalain@univ-brest.fr,Eric.Duvieilbourg@univ-brest.fr
}

\section{Résumé}

Deux types de modèles de propagation de houle en zone côtière fondés soit sur une paramétrisation du spectre : HISWA (Holthuijsen et al., 19895), MIKE21NSW (anonynous, 2002 $2^{1}$ ), soit sur une discrétisation fréquentielle du spectre : TOMAWAC (Benoit, 2002 ${ }^{2}$ ), SWAN (Booij and Holthuijsen, 1999 ${ }^{3}$, Ris et al., $1999^{6}$ ) sont mis en œuvre pour simuler l'évolution spatio-temporelle de l'agitation en baie de Douarnenez durant la campagne à la mer conjointe CNRS-UBOCETMEF EPIES (15 au 24 avril 2005). La validation des modèles s'appuie sur les mesures recueillies par trois bouées houlographiques DATAWELL mouillées en mer d'Iroise (NW de l'île de Sein), à l'entrée de la baie de Douarnenez et face à la plage de Pentrez auxquelles s'ajoute un courantomètre-houlographe VECTOR disposé face à la plage de Sainte Anne-La-Palud. Les capacités prédictives des différents modèles sont évaluées et discutées.

\begin{abstract}
Two types of propagation models in coastal waters based on a (i) parametrization of the spectrum : HISWA (Holthuijsen et al., 1989 $)$ ), MIKE21-NSW (anonymous, $2002^{1}$ ), and a (ii) spectral distribution : TOMAWAC (Benoit, 2002 ${ }^{2}$ ), SWAN (Booij and Holthuijsen, $1999^{3}$, Ris et al. , 19996) are implemented to forecast the spatio-temporal evolution of surface gravity waves in the bay of Douarnenez during the joined-field campaign CNRS-UBO-CETMEF EPIES (from April $15^{\text {th }}$ to April $\left.24^{\text {th }}, 2005\right)$. The models are tested against measurements collected by three waverider buoys DATAWELL moored in the Iroise Sea (NW of Sein island), in the entrance of the bay of Douarnenez, off the beach of Pentrez, and a current-meter VECTOR deployed off the beach of Saint Anne-La-Palud. The predictive capabilities of the various models are estimated and discussed.
\end{abstract}

Mots clés : houle, mesure, modèle numérique, baie de Douarnenez. 


\section{Introduction}

En ingénierie côtière, il est courant d'utiliser des modèles de propagation de houle pour connaître les paramètres de houle dans un secteur littoral à partir de données collectées au large. Ces modèles essentiellement spectraux consistent à résoudre une équation d'évolution de la densité spectrale d'action discrétisée sur plusieurs variables $(\mathrm{x}, \mathrm{y}$, direction $\theta$, fréquence $\omega)$. Au départ stationnaires, ces modèles ont été complétés par des modèles instationnaires grâce aux progrès en matière de puissance de calcul informatique. L'objet de la présente note est d'évaluer quatre de ces modèles, à savoir MIKE21-NSW, HISWA, SWAN et TOMAWAC, en confrontant leurs résultats respectifs à des mesures effectuées dans la zone côtière de la mer d'iroise et de la baie de Douarnenez. L'intérêt d'une prise en compte de la variation de hauteur d'eau due à la marée dans les simulations a également été testé.

\section{Mesures}

Durant la campagne conjointe CNRS-UBO-CETMEF EPIES du 15 au 24 avril 2005 consacrée à la mesure des flux sédimentaires en zone subtidale, trois bouéeshoulographiques DATAWELL et un courantomètre-houlographe VECTOR ont été mouillés en mer d'Iroise et en baie de Douarnenez (voir Figure 1 et Tableau 1).

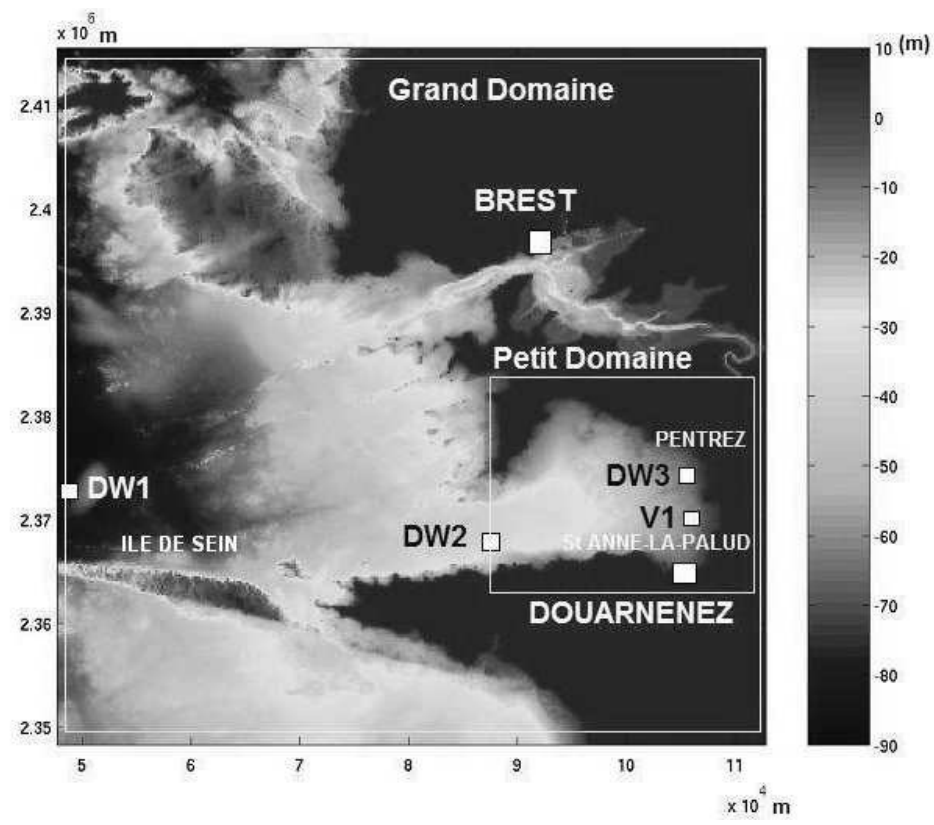

Figure 1 : Localisation des instruments et des domaines de calcul.

La période de mesure fut marquée par une tempête de trois jours qui a généré des vagues au large (DW1) dont la hauteur spectrale significative $\mathrm{H}_{\mathrm{m} 0}$ approchait $4 \mathrm{~m}$ (Figure 2b). L'ensemble des données a été analysé en termes de $\mathrm{H}_{\mathrm{m} 0}$, de période 
du pic d'énergie (Tpic) (Figure 2c) et de direction du pic d'énergie (Dpic) (Figure 2d). La hauteur d'eau prédite par le SHOM montre un marnage passant de 2 à $3 \mathrm{~m}$ durant la période de la tempête (Figure 2a).

\begin{tabular}{|c|c|c|c|}
\hline Site & Profondeur & Type d'instrument & Nom \\
\hline NW de l'île de Sein & $90 \mathrm{~m}$ & Bouée directionnelle & DW1 \\
\hline Sud du Cap de la Chèvre & $40 \mathrm{~m}$ & Bouée directionnelle & DW2 \\
\hline Face à Pentrez & $12 \mathrm{~m}$ & Bouée omni-directionnelle & DW3 \\
\hline Face à Saint Anne-La-Palud & $12 \mathrm{~m}$ & Courantomètre & V1 \\
\hline
\end{tabular}

Tableau 1 : Description de l'instrumentation déployée.
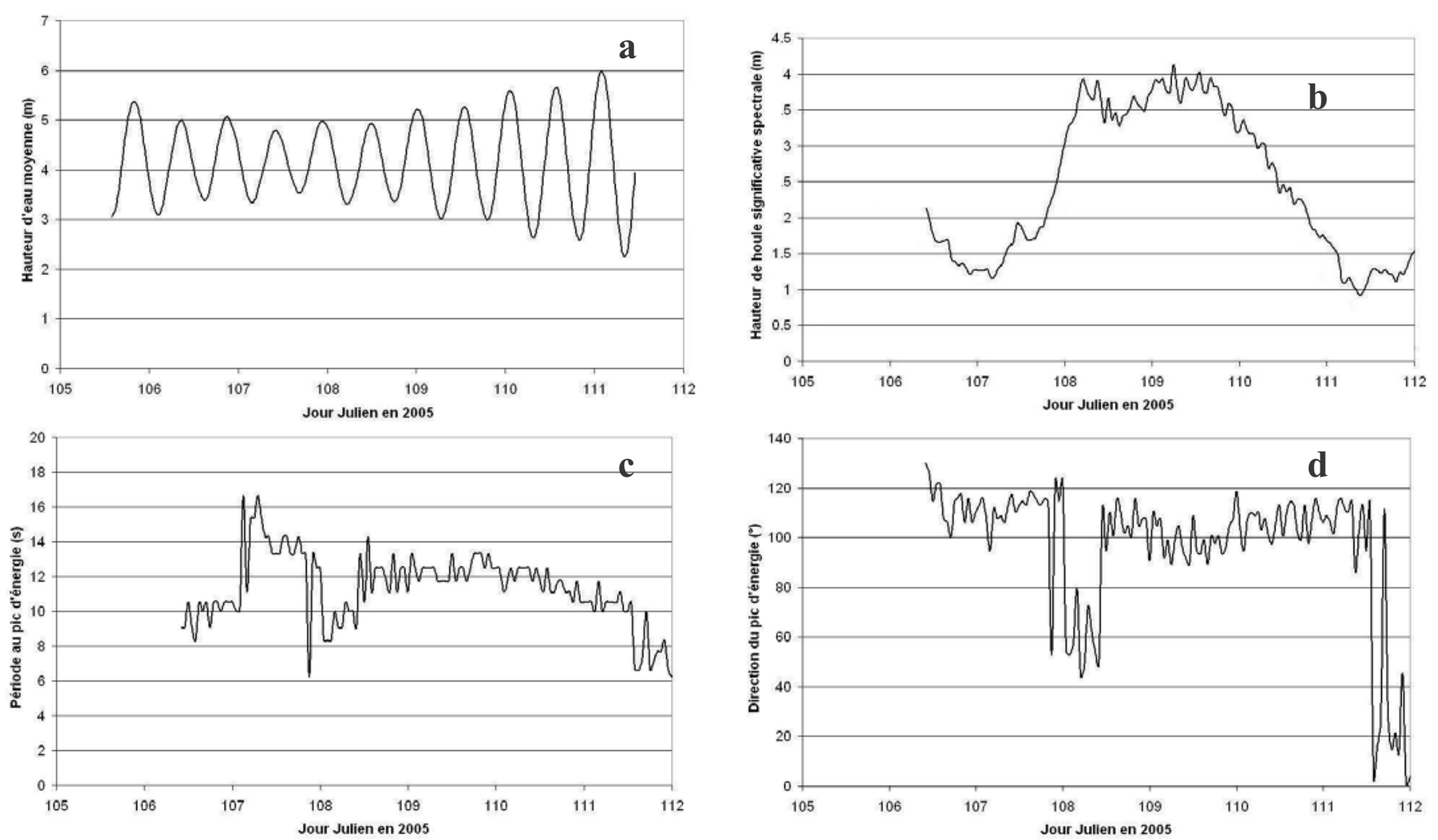

Figure 2 : Hauteur d'eau moyenne prédite par le SHOM (a), hauteur de houle significative spectrale (b), période du pic d'énergie (c) et direction du pic d'énergie (d) mesurées à la bouée DW1.

\section{Modèles utilisés}

Quatre modèles, les uns stationnaires, les autres instationnaires ont été mis en œuvre dans la présente étude.

\section{1. Modèles stationnaires : MIKE 21-NSW et HISWA}

MIKE21-NSW développé au Danish Hydraulics Institute (DHI) (anonymous, $2002^{1}$ ) et HISWA conçu à la Technical University of Denmark (TUD) 
(Holthuijsen et al., 19895) sont des modèles stationnaires qui décrivent le "shoaling », la dissipation par frottement sur le fond et le déferlement. De plus, ils intègrent la réfraction par le courant («blocking effect») et la génération par le vent. Les modèles résolvent une paramétrisation de l'équation de conservation de la densité spectrale d'action d'onde de la houle proposée par Holthuijsen et al. $\left(1989^{5}\right)$ dans le domaine fréquentiel mise en place en introduisant les moments d'ordre 0 et d'ordre 1 du spectre d'action comme des variables dépendantes :

$$
\begin{aligned}
& \frac{\partial\left(c_{g x} m_{0}\right)}{\partial x}+\frac{\partial\left(c_{g y} m_{0}\right)}{\partial y}+\frac{\partial\left(c_{g \theta} m_{0}\right)}{\partial \theta}=S_{0} \\
& \frac{\partial\left(c_{g x} m_{1}\right)}{\partial x}+\frac{\partial\left(c_{g y} m_{1}\right)}{\partial y}+\frac{\partial\left(c_{g} \theta m_{1}\right)}{\partial \theta}=S_{1}
\end{aligned}
$$

dans laquelle : x et y sont les composantes du vecteur direction,

$\theta$ est la direction de propagation,

$\mathrm{m}_{0}$ est le moment d'ordre 0 du spectre d'action,

$\mathrm{m}_{1}$ est le moment d'ordre $1 \mathrm{du}$ spectre d'action,

$\mathrm{c}_{\mathrm{gx}}, \mathrm{c}_{\mathrm{gy}}$ sont les composantes $\mathrm{x}$ et $\mathrm{y}$ de la vitesse de groupe,

$c_{\theta}$ est la vitesse de propagation représentant le changement de

l'action dans la direction $\theta$,

$\mathrm{S}_{0}, \mathrm{~S}_{1}$ sont les termes sources et puits.

Le spectre en fréquence est supposé ne comporter qu'un seul pic. Cette caractéristique rend ces modèles inutilisables pour calculer des états de mers mixtes combinant de la houle et de la mer de vent.

\section{2. Modèles instationnaires : TOMAWAC et SWAN}

Les modèles instationnaires TOMAWAC mis au point au Laboratoire National d'Hydraulique et Environnement (LNHE) / Electricité De France (EDF) (Benoit, $2002^{2}$ ) et SWAN développé à la TUD (Booij and Holthuijsen, $1999^{3}$, Ris et al., $1999^{6}$ ) résolvent respectivement en éléments finis et en différences finies l'équation de conservation du spectre directionnel d'action d'onde de la houle sans effectuer d'approximations sur la nature du spectre (Hasselmann et al., 1973 ${ }^{4}$ ) :

$\frac{\partial N}{\partial t}+\frac{\partial(\dot{x} N)}{\partial x}+\frac{\partial(\dot{y} N)}{\partial y}+\frac{\partial\left(\dot{k}_{x} N\right)}{\partial k_{x}}+\frac{\partial\left(\dot{k_{y}} N\right)}{\partial \dot{k_{y}}}=Q\left(k_{x}, k_{y}, x, y, t\right)$

dans laquelle : t est le temps et $\bullet$ la dérivée temporelle,

$\mathrm{k}_{\mathrm{x}}, \mathrm{k}_{\mathrm{y}}$ sont les composantes du nombre d'onde dans la direction $\theta$, $\mathrm{N}\left(\mathrm{x}, \mathrm{y}, \mathrm{k}_{\mathrm{x}}, \mathrm{k}_{\mathrm{y}}, \mathrm{t}\right)$ est la densité spectro-angulaire d'action d'onde, $\mathrm{Q}$ sont les termes sources et puits de génération et de dissipation. 
Le spectre est décomposé en un nombre fini de fréquences et de directions de propagation. Par rapport aux modèles stationnaires, ces modèles permettent d'inclure les interactions entre quadruplets de fréquences (en grande profondeur) et triplets de fréquences (en faible profondeur).

\section{Conditions de simulation}

Deux domaines de calcul (voir Figure 1) ont été définis pour chacun des quatre modèles :

- un grand domaine « couvrant» la mer d'Iroise «forcé » par la bouée DW1. Les résultats sont comparés aux mesures de la bouée DW2 ;

- un petit domaine «couvrant» la baie de Douarnenez «forcé » par la bouée DW2. Les résultats sont confrontés aux mesures de la bouée DW3 et du courantomètre-houlographe V1.

Les conditions de simulation sont synthétisés dans le Tableau 2. Le "forçage » des modèles s'effectue le long de la frontière ouest par les paramètres d'état de mer $\mathrm{H}_{\mathrm{m} 0}$, Tpic, Dpic et étalement directionnel pour les modèles stationnaires et sous l'approximation d'un spectre de JONSWAP pour les modèles nonstationnaires.

\begin{tabular}{|c|c|c|c|c|}
\hline & MIKE 21NSW & HISWA & TOMAWAC & SWAN \\
\hline $\begin{array}{c}\text { Mode de } \\
\text { fonctionnement }\end{array}$ & $\begin{array}{c}\text { Multi-stationnaire } \\
\text { (i.e. Série de calculs } \\
\text { stationnaires })\end{array}$ & $\begin{array}{c}\text { Multi- } \\
\text { stationnaire }\end{array}$ & Non-stationnaire & Non-stationnaire \\
\hline Mer d'Iroise & $500 \mathrm{~m}$ & $200 \mathrm{~m}$ & $\begin{array}{c}\text { De l'ordre de } 500 \mathrm{~m} \\
\text { (diminuant avec la } \\
\text { bathymétrie) }\end{array}$ & $500 \mathrm{~m}$ \\
\hline $\begin{array}{c}\text { Baie de } \\
\text { Douarnenez }\end{array}$ & $100 \mathrm{~m}$ & $\begin{array}{c}\text { De l'ordre de } 100 \mathrm{~m} \\
\text { (diminuant avec la } \\
\text { bathymétrie) }\end{array}$ & $100 \mathrm{~m}$ \\
\hline
\end{tabular}

Tableau 2 : Conditions de simulation.

\section{Résultats et discussion}

La Figure 3 regroupe l'ensemble des résultats des quatre modèles aux points de mesures DW2 pour la simulation sur la grand domaine, DW3 et V1 pour la simulation sur le petit domaine. Ils se limitent aux simulations effectuées avec un niveau d'eau variable au cours du temps tel que prédit par le SHOM (Figure 2a) car il s'avère que la prise en compte de la variation de la hauteur d'eau conduit à des résultats très proches (de l'ordre du \%) de ceux obtenus avec un niveau moyen de 4,3 $\mathrm{m}$. Ce résultat s'explique par la faiblesse relative du marnage (2 à $3 \mathrm{~m})$ par rapport à la profondeur d'eau au niveau de DW3 et V1 (12m), soit 15 à $25 \%$. 
Session 1 - Hydrodynamique côtière
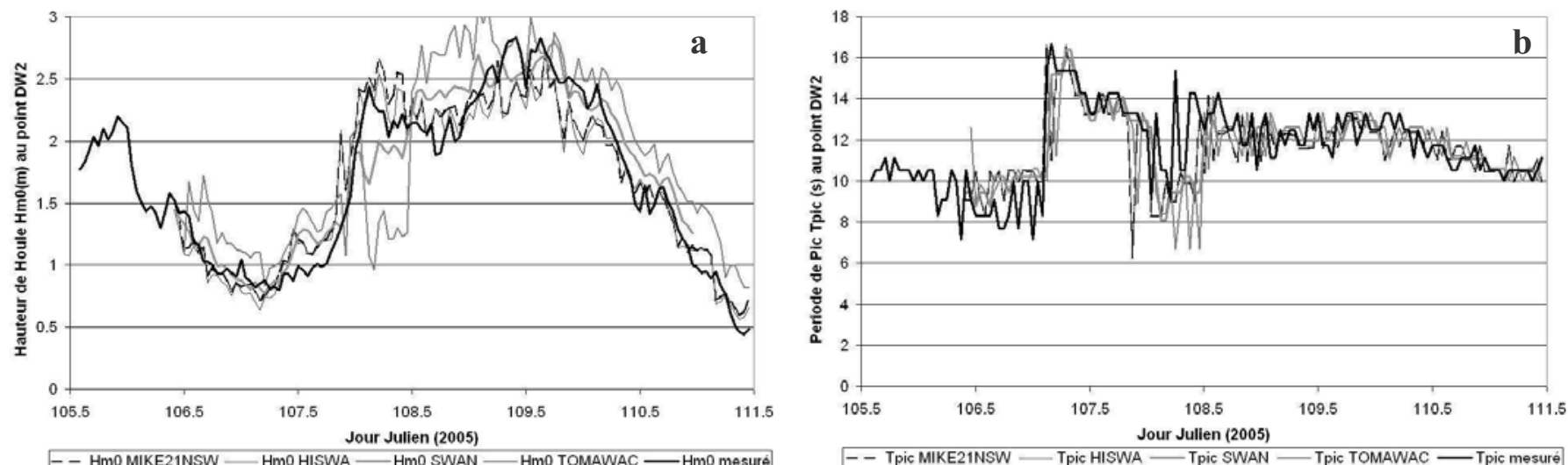

$--\mathrm{HmOMMIKE} 21 \mathrm{NSW}-\mathrm{Hm0HISWA}-\mathrm{HmO}$ SWAN $-\mathrm{Hm0}$ TOMAWAC $-\mathrm{Hm0}$ mesure

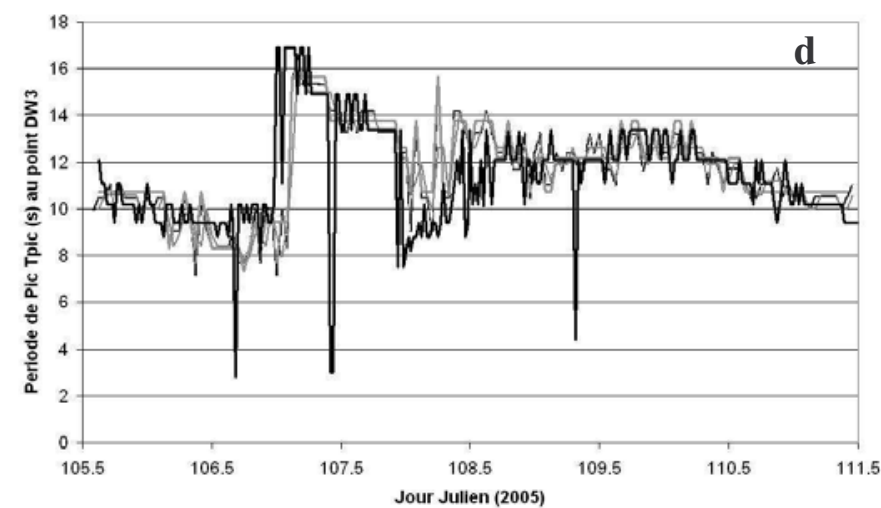

- - Hm0 MIKE21NSW - Hm0 HISWA - Hm0 SWAN — Hm0 TOMAWAC - Hm0 mesure
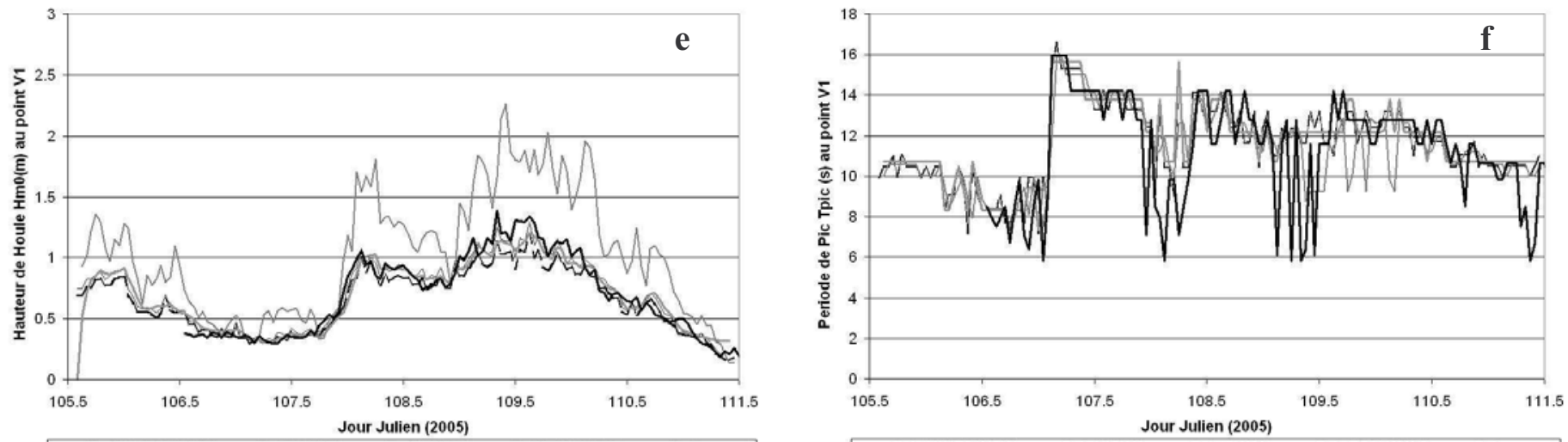

- - Hm0 MIKE21NSW - Hm0 HISWA - Hm0 SWAN - Hm0 TOMAWAC - Hm0 mesuré

- - Tpic MIKE21NSW — Tpic HISWA — Tpic SWAN — Tpic TOMAWAC — Tpic mesure
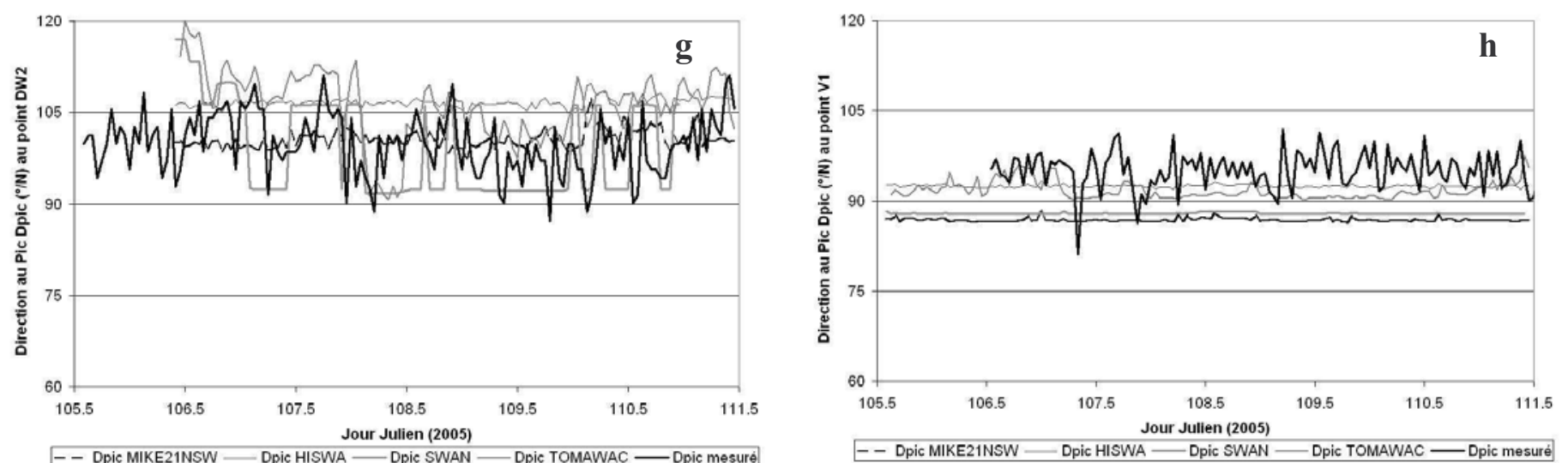

Figure 3 : Comparaisons des résultats des modèles avec les mesures. 
La hauteur de houle significative spectrale $\mathrm{H}_{\mathrm{m} 0}$ (Figure $3 \mathrm{a}$, c et e) est assez bien évaluée par les modèles stationnaires MIKE21-NSW et HISWA aux trois points de mesure (10\% d'erreur au maximum). SWAN prédit correctement $\mathrm{H}_{\mathrm{m} 0}$ en DW2 et $\mathrm{V} 1$, mais la sous-estime de $20 \%$ au point DW3. TOMAWAC peine à reproduire les mesures en les sous-estimant de $40 \%$ en DW3 et en les surestimant de $50 \%$ en V1. La période du pic d'énergie Tpic (Figure 3b, d et f) est quant à elle bien reproduite par tous les modèles. Cependant, celle prédite par MIKE21-NSW et HISWA reste inchangée par rapport au forçage imposée à la frontière ouest . De nature spectrale, les modèles instationnaires SWAN et TOMAWAC prédisent l'évolution de la période du pic de manière un peu plus fidèles aux mesures. Les prédictions des modèles en termes de direction s'inscrivent dans la gamme de valeurs mesurées aux points DW2 et V1, soit entre 90 et $105^{\circ}$ (Figure $3 \mathrm{~g}$ et $\mathrm{h}$ ), mais fluctuent bien moins (quelques degrés) que les mesures.

$\mathrm{Au}$ delà des résultats, signalons enfin que les temps de calcul varient de manière significative d'un modèle à l'autre. Les durées de calcul sur un Pentium IV 3Ghz sont synthétisées au Tableau 3.

\begin{tabular}{|l|l|l|l|l|}
\hline & MIKE21-NSW & HISWA & SWAN & TOMAWAC \\
\hline Niveau marin constant & 1 heure & 1 heure & 10 heures & 9 heures \\
\hline Niveau marin variable & 1 heure & 1 heure & 12 heures & 96 heures \\
\hline
\end{tabular}

Tableau 3: Temps de calcul pour le petit domaine.

\section{Conclusions}

Dans la présente étude, les résultats de quatre modèles numériques ont été comparés à des mesures in-situ. D'un point de vue purement ingénierie, les modèles les plus anciens comme HISWA et MIKE-21NSW semblent mieux approcher les hauteurs significatives spectrales mesurées aux différents points. Avec uniquement les caractéristiques de l'état de mer (représenté par la hauteur significative spectrale de la houle, la période du pic d'énergie, la direction de propagation du pic d'énergie et l'étalement directionnel) donné par les houlographes, de telles modélisations rapides permettent de prévoir de manière satisfaisante la houle en zone subtidale. Cependant, les résultats relatifs à SWAN et TOMAWAC ne sont pas définitifs car toutes les possibilités de ces modèles n'ont pas été exploitées. Afin d'essayer d'améliorer les résultats, des études sont en cours pour notamment (i) intégrer le spectre réel en entrée au lieu d'un spectre de JONSWAP, (ii) utiliser les données de vent et (iii) implémenter une modélisation imbriquée pour «forcer» le long de la frontière ouest du petit domaine avec les résultats issus du grand domaine. 


\section{7. $\underline{\text { Remerciements }}$}

Nous remercions vivement l'ensemble de l'équipage du N/O «Côtes de la Manche » pour leur contribution dans l'acquisition des mesures en baie de Douarnenez lors de la campagne EPIES.

\section{Bibliographie}

1 anonymous (2002). MIKE 21 - Nearshore Spectral Wind-Wave Module Scientific Documentation.

2 Benoit, M. (2002). Logiciel TOMAWAC de modélisation des états de mer en éléments finis. Notice théorique de la version 5.2. Note EDF-R\&DLNHE HP-75/02/065/A.

3 Booij, N. R. C. and Holthuijsen, L. H. (1999). A third generation wave model for coastal regions, part 1, model description and validation. Journal of Geophysical Research, 104(C4):7649-7666.

4 Hasselmann K., Barnett, T. P., Bows, E., Carlson, H., Cartwright, E., Engke, K., Ewing, J. A., Gienapp, H., Hasselmann, D. E., Kruseman, P., Meerburg, A., Müller, P., Olbers, D. J., Richter, K., Sell, W. and Walden, H. (1973). Measurements of wind-wave growth and swell decay during the Joint North Sea Wave Project (JONSWAP). Deutsch Hydrographic Z. Suppl. 12, A8, 1-95.

5 Holthuijsen, L. H., Booij, N. and Herbers, T. H. C. (1989). A Prediction Model for Stationary, Short-crested Waves in Shallow Water with Ambient Currents. Coastal Engineering 13, pp 23-54.

6 Ris, R. C., Holthuijsen, L. H. and Booij, N. (1999). A third-generation wave model for coastal regions, part 2, verification. Journal of Geophysical Research, 104(C4):7667-7681. 